\title{
Resonancia magnética biparamétrica en la biopsia de precisión prostática por ultrasonido endorrectal
}

\section{Bi-parametric magnetic resonance in prostate biopsy by endorectal ultrasound}

Juan F. García-Quintanilla*, Omar Sanchez-Frias, Samuel I. Espinoza, Eustorgio Ruiz-Chapa, Luis A. Zavala, Gerardo Martínez-Sáenz y Francisco J. Ruiz

Centro de Radiodiagnóstico SC, Monterrey, Nuevo León, México

\section{RESUMEN}

La resonancia magnética biparamétrica se ha convertido en una herramienta importante en la detección de sospecha de cáncer de próstata como ha sido comprobado en este reporte. Este y otros informes recientes evidencian el cambios de enfoque, en lugar de biopsias múltiples aleatorias a biopsias de próstata dirigidas a lesiones diana.

Palabras clave: Próstata. RM biparamétrica. Biopsia endorrectal. DWI. Cáncer de próstata. IRM prebiopsia.

\section{ABSTRACT}

Bi-parametric magnetic resonance imaging has become a mayor tool in the detection of suspect prostate cancer as been proven in the present report. This and other recent reports should change the approach instead multiple random biopsies for target prostate biopsies.

Key words: Prostate. Bi-parametric. MRI. Endorectal biopsies. DWI. Prostate cancer. Pre-biopsy MRI. 


\section{INTRODUCCIÓN}

Desde el inicio de la década de los noventa, el uso del antígeno prostático específico (APE) en el escrutinio y búsqueda del cáncer de próstata llevó al siguiente paso, que fue la implementación de la toma de biopsia que, desde la punción directa transperineal o vía transrectal por palpación o guiada por ultrasonido endorectal (USER), ha prevalecido hasta la fecha como estudio confirmatorio histológico del carcinoma de la próstata $(\mathrm{CP})^{1,2}$.

Múltiples técnicas han sido descritas y utilizadas desde el USER con toma de biopsia múltiple por cuadrantes, como sextantes, muestreo aleatorio por zonas hipervasculares por Doppler color o biopsia múltiple por saturación, con muy diversos resultados en cuanto a positividad: el acierto para CP ronda entre el 40 y el 50\%, dependiendo de las series y del abordaje utilizado ${ }^{2}$. La imagen por resonancia magnética (IRM) en la próstata, desde principios de la década de los noventa, se ha utilizado en muy diversas formas, desde el uso de antenas o dispositivos endorrectales, hasta antenas de superficie Sensitivity encoding, Philips (SENSE) y conceptos multiparamétricos que incluyen imagen anatómica potenciada en T1 y T2, medios de contraste basándose en el gadolinio, espectroscopia y difusión (DWI)2, técnicas con las cuales se ha hecho evidente la visualización implícita de lesiones altamente sospechosas de neoplasia. El adenocarcinoma se origina de la periferia prostática -la glándula propiamente dicha-, sin embargo, con el paso de los años y la disminución de testosterona, la hipertrofia del lóbulo prostático central propicia la invasión y distorsión periférica y, al perderse el límite anatómico entre ambas zonas, encontramos neoplasia «sin fronteras», es decir, centro y periferia como una gran área proclive a desarrollar cáncer, área también conocida como zona de transición $^{3-4}$. Dado el costo, morbilidad y dudoso acierto de las biopsias múltiples por USER en la detección de CP (50\% de acierto en las mejores manos $^{2-5}$ ), el uso de IRM multiparamétrica ha permitido la identificación de neoplasia en forma expedita ${ }^{5,6}$. Nosotros hemos implementado el uso de la IRM con antena de superficie-SENSE-, sin usar antena endorrectal, y después de varios años de utilizar IRM multiparamétrica, encontramos que dos parámetros -secuencia T2 y DWI- son suficientes para demostrar pequeñas lesiones, lo que hace mucho más fácil su identificación por USER, con muchas menos muestras por true-cut $y$, por ende, disminuyendo la morbilidad y el costo del procedimiento.

\section{MATERIAL Y MÉTODOS}

De enero del 2015 a diciembre del 2016, a 200 pacientes se les practicó una biopsia guiada por USER, previa IRM biparamétrica. Las edades de los pacientes fluctuaron entre los $47 \mathrm{y}$ los 78 años. Todos fueron referidos por médicos urólogos y todos por elevación constante del APE $(5 \mathrm{ng} / \mathrm{ml}$ hasta $120 \mathrm{ng} / \mathrm{ml})$. Previo a la biopsia y si no existía contraindicación, como uso de marcapaso o claustrofobia, y previa firma de consentimiento informado, se les practicó un estudio de IRM biparamétrico de la pelvis, que consiste en secuencias T2 axial y coronal, así como secuencias en DWI en valores b 0 y b 1,500, en un sistema Philips Ingenia $1.5 \mathrm{~T}$ y utilizando una antena receptora de superficie tipo SENSE (Tabla 1). Posteriormente y previo a la biopsia, las imágenes 
Tabla 1. Protocolo de adquisición de imágenes en secuencia de T2 en plano axial y en secuencia de difusión en valor bajo $y$ alto

\begin{tabular}{|l|c|c|}
\hline & T2 & DWI b 0 - b 1,500 \\
\hline Field of view (FOV) & $170 \times 170$ & $180 \times 200$ \\
\hline $\begin{array}{l}\text { Matriz de } \\
\text { adquisición }\end{array}$ & $152 \times 118$ & $68 \times 59$ \\
\hline $\begin{array}{l}\text { Tiempo de } \\
\text { repetición (Tr) }\end{array}$ & 5992 & 4000 \\
\hline $\begin{array}{l}\text { Tiempo de eco (Te) } \\
\text { Flip angle }\end{array}$ & 120 & 110 \\
\hline $\begin{array}{l}\text { Grosor de corte } \\
\text { Matriz de } \\
\text { reconstrucción }\end{array}$ & $30 \mathrm{~mm}$ & 30 \\
\hline $\begin{array}{l}\text { Tiempo de } \\
\text { adquisición }\end{array}$ & $2 \mathrm{~min} 05 \mathrm{~s}$ & $144 \times 144$ \\
\hline
\end{tabular}

fueron evaluadas por dos médicos radiólogos en búsqueda de los siguientes hallazgos: nódulo o zona hipointensa en la periferia o zona de transición prostática en secuencia T2 que mostrara restricción en DWI, exclusivamente en valores altos b 1,500 (Figs. 1 y 2). La toma de decisión llevó 10 minutos en promedio en cuanto a búsqueda de nódulos o áreas de sospecha, y a los pacientes que no se les encontró imagen con estas características no se les practicó biopsia y se les refirió al médico tratante para seguimiento clínico y APE. Se seleccionaron 200 pacientes que cumplieron la totalidad de los requerimientos del protocolo de IRM en cuanto a la visualización de lesiones de sospecha y previa preparación con antibióticos y enema evacuante. Se les practicó biopsia dirigida por USER, con un sistema Philips IU 22 con transductor endocavitario, ubicando los hallazgos por IRM al USER, en forma cognitiva-anatómica, y se obtuvieron entre dos a cinco muestras por paciente, tres muestras en promedio,

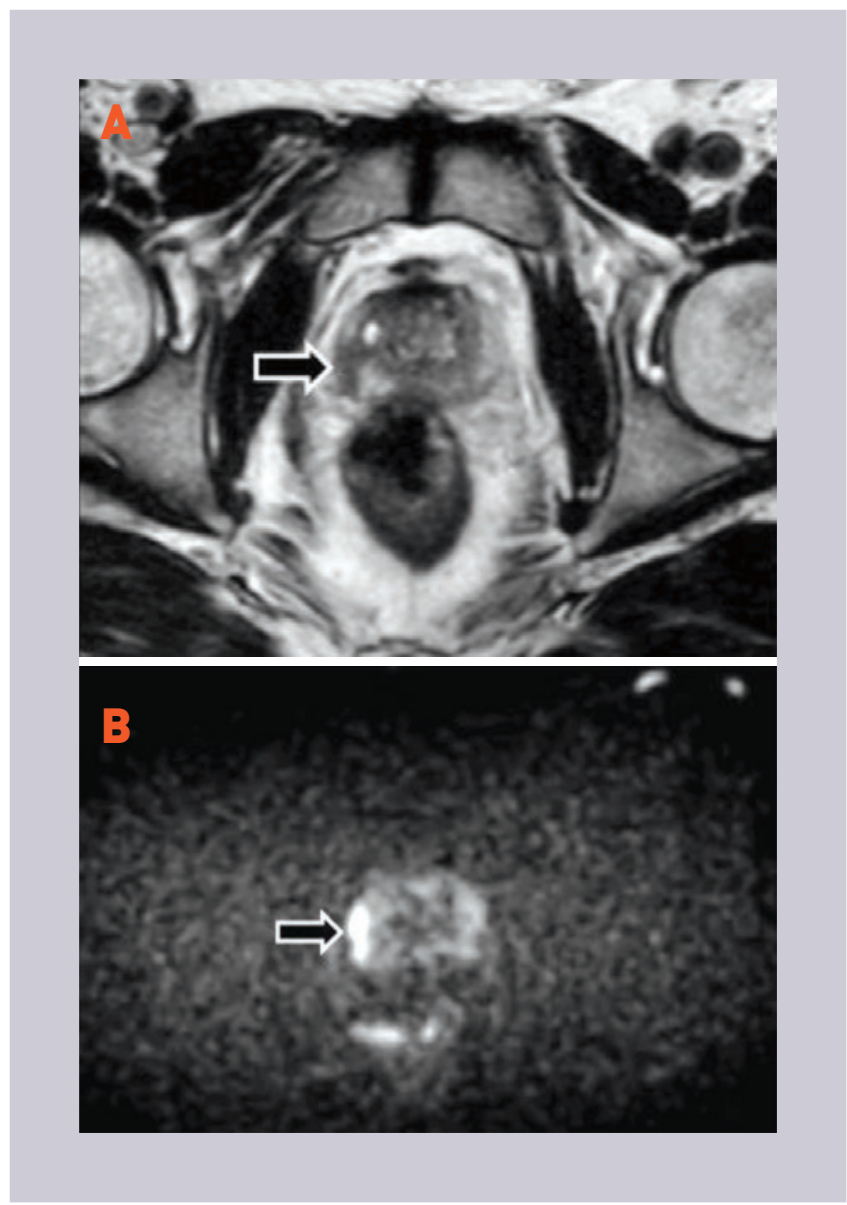

Figura 1. A: secuencia T2 en plano axial que muestra un nódulo hipointenso (flecha) en la zona periférica derecha (TR5990, TE 120). B: secuencia de difusión DWI (b 1,500$)$ que muestra una zona de restricción focal (flecha), que se corresponde con el nódulo observado en la secuencia de T2 (A).

con aguja true-cut del n. ${ }^{\circ} 18$, distribuidas de la siguiente forma: dos o tres muestras de la zona seleccionada de sospecha por IRM y una o dos muestras aleatorias en el lóbulo o zona opuesta- en espejo- a las muestras obtenidas en la zona de sospecha -lóbulo contrario-.

\section{RESULTADOS}

De los 200 pacientes con áreas de sospecha por IRM que se les practicó biopsia por USER, se obtuvo histología positiva para adenocarcinoma 

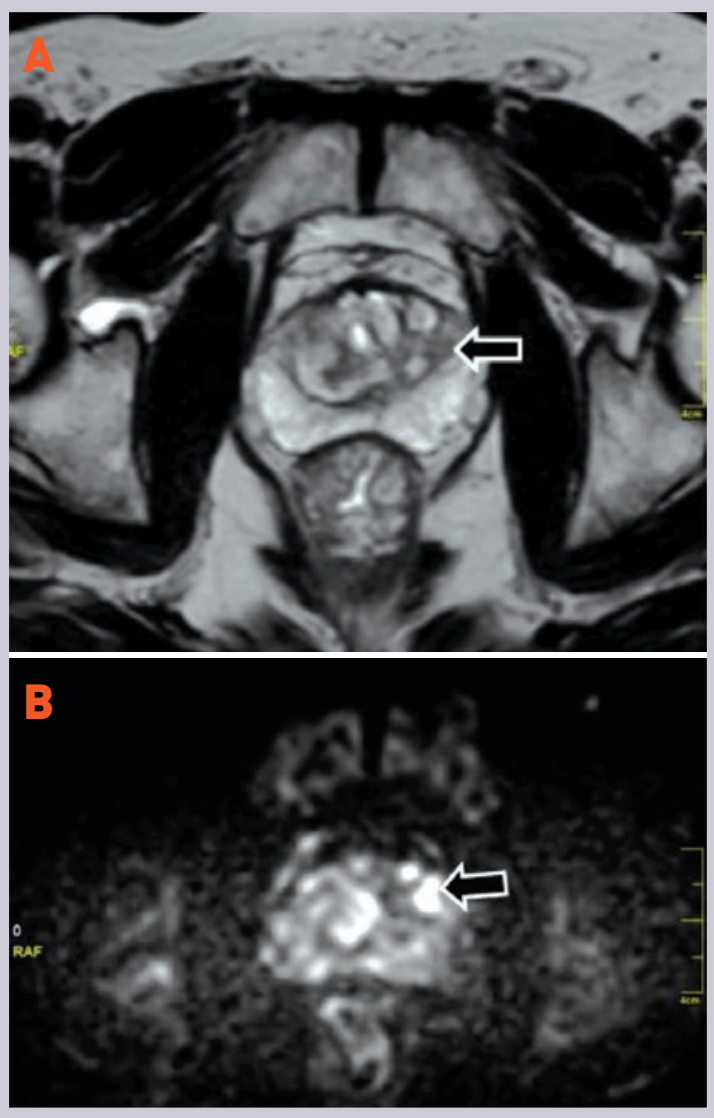

Figura 2. A: secuencia T2 en plano axial con nódulo hipointenso en la zona de transición izquierda (flecha) (TR5990, TE 120). B: Secuencia DWI b 1,500 que muestra restricción del nódulo observado en $A$.

prostático en el 87\% (174 pacientes), con un promedio de tres muestras por paciente. Solo en el 1\% (2 pacientes) resultó positiva la muestra aleatoria -en espejo- para CP. En 24 pacientes, la biopsia reportó solamente hallazgos de prostatitis o hipertrofia glandular.

\section{DISCUSIÓN}

La rutina de la IRM multiparamétrica en la búsqueda de patología prostática incluye imágenes T2 en cortes de $3 \mathrm{~mm}$, DWI en valores mayores de 1,000 seg $/ \mathrm{mm}^{2}$, imagen contrastada dinámica y espectroscopia. En general, la mayoría de los CP tienden a localizarse en la periferia prostática y típicamente son nódulos o áreas bien definidas e hipointensas en T2, con alta restricción a la DWI en valores altos y valores bajos en los parámetros de coeficiente apparent diffusion coefficient (valores ADC) ${ }^{6-8}$. Las lesiones malignas en zonas de transición tienden a tener bordes menos nítidos, sin cápsula y con infiltración al estroma vecino. Una característica que hemos encontrado es que el tejido inflamatorio o no neoplásico tiende a restringir en valores DWI bajos (b 0), a diferencia del tejido neoplásico que lo hace casi exclusivamente en valores altos. Estos hallazgos que son concordantes con publicaciones y comunicados recientes ${ }^{9-11}$.

Quizá los tipos de restricción por DWI estén ligados a la escala Gleason; sin embargo, es algo que queda por validar, dado que la escala histológica de Gleason es un parámetro de celularidad -a mayor celularidad mayor escala de atipia histológica-. El movimiento de agua intra-extracelular a través de la membrana celular se limita enormemente -como lo es en las neoplasias- $y$, por ende, existe caída en la señal dependiente de T2 y mayor restricción de la señal por IRM en DWI, principalmente en valores altos ${ }^{9,10}$, es decir, a mayor celularidad, mayor escala Gleason y, por consecuencia, mayor restricción por DWI. Hemos comprobado que las biopsias dirigidas sobre estas áreas son mucho más certeras en positividad de CP, y con muchas menos muestras que los procedimientos de biopsia por mapeo, aleatorios o por saturación, los cuales llevan en promedio 12 muestras, e incluso hasta 24 muestras en la llamada biopsia ampliada, con un acierto para positividad de CP no mayor al 
45\% ${ }^{4-11}$. El uso como protocolo de otros parámetros en IRM, como son la espectroscopia y medios de contraste, a nosotros no nos ha sido útil, principalmente por concepto de tiempo de magneto y costo. Hemos encontrado que la relación de secuencias anatómicas por IRM T2 y fisiológicas DWI en valores altos resultan en una combinación rápida y efectiva para la localización de zonas sospechosas que deban ser sometidas a biopsia, hecho que ya ha sido validado por otros grupos ${ }^{12}$. Hemos encontrado también la forma de ubicar las lesiones en USER vistas por IRM por medio cognitivo (anatómico) sin utilizar aditamentos de ubicación de lesiones, como es la fusión digital imaging and communication in medicine (DICOM) de imágenes. El utilizar IRM como preámbulo o escrutinio de lesiones con sospecha de atipia en próstata nos ha permitido disminuir en forma considerable el número de muestras (true-cut) y, por consiguiente, la disminución en morbilidad, además de elevar casi al doble la certeza histológica de neoplasia $(87 \%$ de positividad para $\mathrm{CP}$ ) con respecto a otros procedimientos que no usan IRM como guía1113. También ha resultado sumamente eficiente la IRM biparamétrica en ubicar lesiones de difícil valoración al USER o a la exploración física digital, como son los nódulos en el ápex, lesiones retropúbicas o lesiones en próstatas hipertróficas.

Un reto actual es llevar a biopsia nódulos de sospecha en el mismo equipo de RM, procedimiento que en la actualidad resulta caro y laborioso, pero que deberá llevar a menos resultados falsos negativos y elevar el diagnóstico histológico de certeza ${ }^{14-15}$. Algo característico y bien documentado en la periferia prostática son las zonas inflamatorias (prostatitis) o áreas en «cuña» con bordes geográficos y con una manifestación hipointensa en secuencia T2, con un comportamiento restrictivo a DWI, tanto en valores b bajos como b altos, así como valores ADC altos, zonas de prostatitis que permiten ser diferenciadas del CP, el cual, como ha sido documentado en este trabajo, restringe únicamente en valores b DWI altos. El uso de medio de contraste (gadolinio) ha resultado de utilidad en pacientes con historial de biopsias múltiples, pacientes en los que las zonas de la cicatriz por biopsias previas pudieran resultar confusas en las secuencias biparamétricas (T2 o DWI). Este medio de contraste proporciona una relativa información en cuanto al realce de lesiones sospechosas de neoplasia ${ }^{16,17}$.

\section{CONCLUSIÓN}

Para nosotros, ha resultado evidente que el uso de la IRM biparamétrica previa a la biopsia por USER ha incidido favorablemente en un mucho mayor acierto de $\mathrm{CP}$, con muchas menos muestras que los procedimientos de biopsia aleatorios, por sextantes o por saturación, y creemos que esta información debe incidir en la forma actual de diagnosticar el CP.

\section{RESPONSABILIDADES ÉTICAS}

Protección de personas y animales. Los autores declaran que para esta investigación no se han realizado experimentos en seres humanos ni en animales.

Confidencialidad de los datos. Los autores declaran que han seguido los protocolos de su centro de trabajo sobre la publicación de datos de pacientes. 


\section{Derecho a la privacidad y consentimiento informado. Los autores han obtenido el con- sentimiento informado de los pacientes y/o sujetos referidos en el artículo. Este documen- to obra en poder del autor de correspondencia.}

\section{BIBLIOGRAFÍA}

1. Polascik TJ, Oesterling JF, Partin AW. Prostate specific antigen : A decade of discovery-what we have learned and where we are going. J Urol. 1999;162: 293-306.

2. Lawton C. Prostate Cancer: Improving the flow of research. Radiology. 2018;287:5-9.

3. Zakian KL, Eberhardt S, Hricak H, Shukla-Dave A, Kleinman S, Muruganandham M. Transition zone prostate cancer: metabolic characteristics at 1H MR spectroscopic imaging-initial results. Radiology. 2003;229:241-7.

4. Akin O, Sala E, Moskowitz CS, Kuroiwa K, Ishill NM, Pucar D, et al. Transition zone prostate cancers: features, detection, localization and staging at endorectal MR imaging. Radiology. 2006;239:784-92.

5. Bratan F, Melodelima C, Souchon R, Hoang Dinh A, Mège-Lechevallier F, Crouzet $S$, et al. How accurate is multiparametric MR imaging in evaluation of prostate cancer volume? Radiology. 2015;275:144-54

6. Niaf E, Lartizien C, Bratan F, Roche L, Rabilloud M, Mège-Lechevallier F, et al. Prostate focal peripheral zone lesions: Characterization at multiparametric MR imaging-influence of computer aided diagnosis system. Radiology. 2014;271:761-9.
7. Bloch BN, Furman-Haran E, Helbich TH, Lenkinski RE, Degani H, Kratzik C,et al. Prostate Cancer: accurate determination of extracapsular extension with high spatial resolution dynamic contrast-enhancement and T2-weighted MR imaging-initial results. Radiology. 2007;245:176-85.

8. Borofsky S, George AK, Gaur S, Bernando M, Greer MD, Mertan FV, et al. What are we missing? False-negative cancer at multiparametric imaging of the prostate. Radiology. 2018;286:186-95.

9. Haider MA, van der Kwast TH, Tanguay J, Evans AJ, Hashmi AT, Lockwood $\mathrm{G}$, et al. Combined T2-weighted and diffusion-weighted MRI for localization of prostate cancer. AJR. 2007;189:323-8.

10. Canals M, Salas D, Canals A, Siches I, Van Cauwelaert R, Barros R. Valor de la secuencia de difusión en el estudio de cáncer de próstata en pacientes con APE elevado. Rev Chilena de Rad. 2010;16:35-42.

11. Rodriguez A, Gonzalez A, Pita S, Suárez G, Bonelli C, Lorenzo J, et al. Diagnóstico del cáncer de próstata mediante biopsia ampliada de 24 cilindros. Actas Urol Esp. 2015;29:37-42.

12. Hricak H. Imaging prostate cancer, detection/localization using T2WI and DWI. European Congress of Radiology. Vienna, 2018.

13. Lamb BW, Tan WS, Rehman A, Nessa A, Cohen D, O'Neil J, et al. Is pre-biopsy MRI good enough to avoid prostate biopsy? A cohort study over a 1 year period. Clin Genitourin Cancer. 2015;13:512-7.

14. Vilanova JC, Comet J, Garcia- Figueras R, Barceló J, Boada M. Utilidad de la RM en el cáncer de próstata. Radiología. 2010;52(6 ):513-24.

15. Ghai S, Trachtenberg J. MRI-guided biopsies and minimally invasive therapy for prostate cancer. Indian J Urology. 2015;31: 33-42.

16. Fütterer JJ, Heijmink SW, Scheenen TW, Veltman J, Huisman HJ, Vos P, et al. Prostate cancer localization with dynamic contrast enhancement MR imaging and proton MR spectroscopic imaging. Radiology. 2006;241:449-58.

17. Rosenkrantz A, Babb J, Taneja S,Ream JM. Proposed adjustments to PI-RADS version 2 decision rules: Impact of prostate cancer detection. Radiology. 2017;283:119-29. 